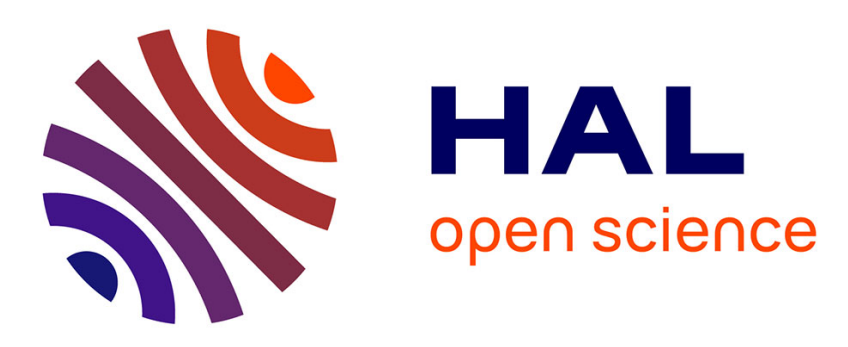

\title{
Size invariance of the granular Rayleigh-Taylor instability
}

Jan Ludvig Vinningland, Øistein Johnsen, Eirik Grude Flekkøy, Renaud Toussaint, Knut Jørgen Måløy

\section{To cite this version:}

Jan Ludvig Vinningland, Øistein Johnsen, Eirik Grude Flekkøy, Renaud Toussaint, Knut Jørgen Måløy. Size invariance of the granular Rayleigh-Taylor instability. Physical Review E: Statistical, Nonlinear, and Soft Matter Physics, 2010, 81 (4), 10.1103/PhysRevE.81.041308 . hal-02377790

\section{HAL Id: hal-02377790 \\ https://hal.science/hal-02377790}

Submitted on 24 Nov 2019

HAL is a multi-disciplinary open access archive for the deposit and dissemination of scientific research documents, whether they are published or not. The documents may come from teaching and research institutions in France or abroad, or from public or private research centers.
L'archive ouverte pluridisciplinaire HAL, est destinée au dépôt et à la diffusion de documents scientifiques de niveau recherche, publiés ou non, émanant des établissements d'enseignement et de recherche français ou étrangers, des laboratoires publics ou privés. 


\title{
Size invariance of the granular Rayleigh-Taylor instability
}

\author{
Jan Ludvig Vinningland, ${ }^{1,2, *}$ Øistein Johnsen, ${ }^{1}$ Eirik G. Flekkøy, ${ }^{1}$ Renaud Toussaint, ${ }^{3}$ and Knut Jørgen Måløy ${ }^{1}$ \\ ${ }^{1}$ Advanced Materials and Complex Systems, Department of Physics, University of Oslo, P.O. Box 1048, 0316 Oslo, Norway \\ ${ }^{2}$ Physics of Geological Processes, Department of Physics, University of Oslo, P.0. Box 1048, 0316 Oslo, Norway \\ ${ }^{3}$ Institut de Physique du Globe de Strasbourg, CNRS, Université Louis Pasteur, 5 rue Descartes, 67084 Strasbourg Cedex, France \\ (Received 3 September 2008; revised manuscript received 18 February 2010; published 29 April 2010)
}

\begin{abstract}
The size scaling behavior of the granular Rayleigh-Taylor instability [J. L. Vinningland et al., Phys. Rev. Lett. 99, 048001 (2007)] is investigated experimentally, numerically, and theoretically. An upper layer of grains displaces a lower gap of air by organizing into dense fingers of falling grains separated by rising bubbles of air. The dependence of these structures on the system and grain sizes is investigated. A spatial measurement of the finger structures is obtained by the Fourier power spectrum of the wave number $k$. As the size of the grains increases the wave number decreases accordingly which leaves the dimensionless product of wave number and grain diameter, $d k$, invariant. A theoretical interpretation of the invariance, based on the scaling properties of the model equations, suggests a gradual breakdown of the invariance for grains smaller than $\sim 70 \mu \mathrm{m}$ or greater than $\sim 570 \mu \mathrm{m}$ in diameter.
\end{abstract}

DOI: 10.1103/PhysRevE.81.041308

PACS number(s): 81.05.Rm, 47.20.Ma, 47.11.-j, 45.70.Qj

\section{INTRODUCTION}

Granular materials are the basis of abundant industrial and natural processes and an integral part of our everyday life. Dry granular flows have been widely studied over the past twenty years [1], but the study of granular flows where the interstitial fluid plays an important role is still in its infancy. Many natural phenomena pertaining to such granular/fluid flows are active topics of current research, e.g., sedimentation $[2,3]$, erosion and river evolution [4], underwater avalanches and turbidites [5], and soil fluidization during earthquakes [6]. Industrial processes, such as pneumatic transport, fluidized beds, catalytic cracking $[1,7,8]$ would also benefit from advances in granular/fluid flow research.

The granular Rayleigh-Taylor instability arises when a closed, vertical Hele-Shaw cell, partially filled with fine grains and air at atmospheric pressure, is rapidly rotated to bring the dense packing of grains above a layer of air. Shortly after the rotation fine fingers of falling grains emerge from the initially flat grain-air interface. These fine fingers subsequently develop into coarser finger structures separated by bubbles of air. In contrast to the classical Rayleigh-Taylor instability [9] the coarsening process observed in the granular case will right from the start bring about a reduction in the number of fingers and a corresponding increase in the size of the bubbles. New fingers will form in the center of the rising bubbles as they reach a certain width, and the characteristic size of the structures is thus maintained at a stable value.

This granular instability is previously studied both numerically and experimentally $[10,11]$, using the same model and setup as in the present paper, with the conclusion that the two competing mechanisms, one producing finer scales, the other producing coarser scales, are well reproduced by the numerical model.

In contrast to the gas-grain instability reported here, Völtz et al. studied a liquid-grain instability experimentally and

\footnotetext{
*janlv@fys.uio.no
}

theoretically [12]. The liquid-grain instability bears a strong resemblance to the classical instability for two liquids with almost overlapping dispersion relations. The gas-grain instability, however, displays a quite different behavior with a coarsening process active from the beginning and a counteracting refinement process that stabilizes the average size of the bubbles.

The purpose of the present investigation is to study the variations in the flow structures under change of spatial scale of both the grains and the Hele-Shaw cell. Size invariance is not commonly studied for granular materials. Indeed, granular flows are quite sensitive to initial preparation and external perturbations, so that systematic grain and system size changes are delicate studies. In the present paper, using the complementarity of experimental and numerical techniques, we perform a systematic study of how the structures formed by the granular Rayleigh-Taylor instability responds to a rescaling of the system and grain sizes.

The experimental, numerical, and theoretical results presented all indicate a size invariance of the finger-bubble structures. However, the theoretical analysis predicts a breaking of the invariance if the grains are too small or too large. The simulations use grains ranging from 70 to $490 \mu \mathrm{m}$ in diameter, and the experiments use grains ranging from 80 to $570 \mu \mathrm{m}$ in diameter. Consistent data collapses are obtained from these results and demonstrate a fairly robust invariance within the given limits.

The simulations and experiments presented in Ref. [11] were performed with identical parameters to provide an assessment of the numerical model. In the present paper we present experimental and numerical results obtained in a new parameter regime not explored in previous publications. However, in this paper the parameters of the experiments and simulations are no longer identical, due to less emphasis on model assessment. In particular, the simulations and experiments are carried out using slightly different grain densities. The scale invariance of the granular Rayleigh-Taylor invariance was first acknowledged in Ref. [10]. The current paper provides a more elaborate discussion of this invariance in- 
TABLE I. Listing of the diameters of the grains and the dimensions of the Hele-Shaw cells used in the experiments.

\begin{tabular}{lcccc}
\hline $\begin{array}{l}\text { Diameter } \\
(\mu \mathrm{m})\end{array}$ & 80 & 140 & 230 & 570 \\
\hline Width $(\mathrm{mm})$ & 31 & 56 & 91 & 200 \\
Height $(\mathrm{mm})$ & 61 & 86 & 141 & 305 \\
Depth $(\mathrm{mm})$ & 1.0 & 1.7 & 2.3 & 5.4 \\
\hline \hline
\end{tabular}

cluding results from experiment, simulation and theory not presented in [10].

The paper is organized as follows. A presentation of the experimental setup and implementation is given in the next section. The numerical model is briefly presented in Sec. III, and the results, together with the data analysis, are presented and discussed in Sec. IV. The theoretical basis of the size invariance, based on the scaling properties of the model equations, is presented in Sec. V. Section VI presents numerical data obtained to check for possible container size effects. Finally, a conclusion and summary are given in Sec. VII.

\section{EXPERIMENT}

The experimental setup is the same as was used in Refs. $[10,11]$ and will only be presented briefly here. Four HeleShaw cells of different sizes are partially filled with monodisperse polystyrene beads and air at atmospheric pressure. The sizes of the cells scale proportionally with the diameter of the grains they contain, see Table I. The cells are filled with Dynoseeds polystyrene spheres of density $\rho_{g}=1.05 \mathrm{~g} / \mathrm{cm}^{3}$ (manufactured by Microbeads [13]). The cells are individually mounted on a hinged bar and rapidly rotated to an upright position, see Fig. 1. Images of the falling grains are recorded by a high speed digital camera (Photron Fastcam-APX 120K) at a rate of 500 frames per second.

The humidity in the laboratory was kept at about $30 \%$ during the assembling of the cells, and the grains were exposed to the air in the laboratory for some time before the

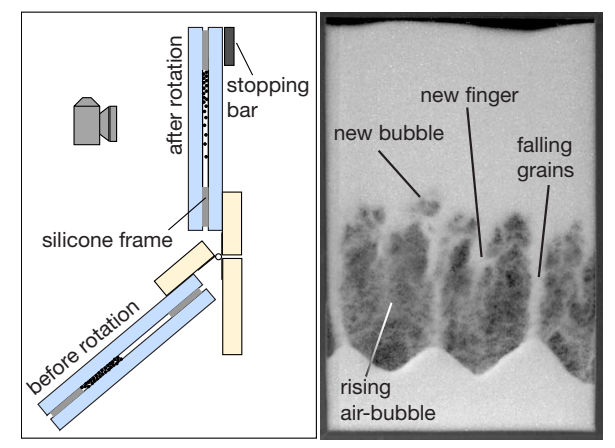

FIG. 1. (Color online) (left) Side view of the experimental setup with the initial and final cell positions superimposed. (right) Typical snapshot from an experiment using grains of $230 \mu \mathrm{m}$ in diameter in a cell that is $91 \times 141 \times 2.3 \mathrm{~mm}$. filling. This is to prevent clustering of the grains due to cohesion, arising from capillary bridges, or electrostatic forces.

The experimental cells are somewhat taller than the numerical cells to account for the grains that settle during the rotation. The horizontal filling procedure of the cells makes it difficult to control the filling fraction, and it may vary slightly between the four cells. During the assembling of the cells the TS 500-53 beads was presumed to have a diameter of $500 \mu \mathrm{m}$. However, an analysis performed by Microbeads shows that the mean diameter of these grains is in fact $570 \mu \mathrm{m}$. This explains the slight discrepancy between the width of the cell and the diameter of the grains in this case.

\section{SIMULATION}

The numerical model has proved to be very consistent in reproducing experimentally observed structures in granular flows and instabilities in the regime of low Reynolds numbers [14-17]. The theoretical basis and derivation of the model are given in Refs. [14,15] and a description of the current implementation is given in Ref. [11]. However, a brief outline of the model will be presented here for completeness.

The granular phase is modeled as discrete, rigid spheres that collectively constitute a deformable porous medium. The pressure drop associated with the air flowing through the granular medium is given by a local Darcy's law [18] with the Carman-Kozeny relation for the permeability $\kappa[19]$. The continuum gas phase is described solely by its pressure $P$. The velocity field of the gas may be obtained from the pressure gradient via Darcy's law. In order to provide a continuum description of the pressure $P(x, y)$ on a grid $(x, y)$, the continuum variables, solid fraction $\rho(x, y)$, and granular velocity $\mathbf{u}(x, y)$ need to be calculated. This is done by a linear smoothing function $s\left(\mathbf{r}-\mathbf{r}_{0}\right)$ expressed mathematically as [14]

$$
s\left(\mathbf{r}-\mathbf{r}_{0}\right)= \begin{cases}\left(1-\frac{\Delta x}{l}\right)\left(1-\frac{\Delta y}{l}\right) & \text { if } \Delta x, \Delta y<l \\ 0 & \text { otherwise }\end{cases}
$$

where $\mathbf{r}(x, y)$ is the position of the grain, $\mathbf{r}_{0}\left(x_{0}, y_{0}\right)$ is the position of the grid node, $\Delta x=\left|x-x_{0}\right|$ and $\Delta y=\left|y-y_{0}\right|$ are the relative distances, $l$ is the lattice constant, and $\Sigma_{k} s\left(\mathbf{r}-\mathbf{r}_{k}\right)=1$ with $k$ indexing the four neighboring sites of a grain positioned at $\mathbf{r}$.

From the continuity equations of air mass and grain mass, using the average velocity of the air in the porous matrix (i.e., $\mathbf{u}_{\text {Darcy }} / \phi$ ), a pressure equation is derived, see $[14,15]$,

$$
\phi\left(\frac{\partial P}{\partial t}+\mathbf{u} \cdot \boldsymbol{\nabla} P\right)=\boldsymbol{\nabla} \cdot\left(P \frac{\kappa(\phi)}{\mu} \boldsymbol{\nabla} P\right)-P \boldsymbol{\nabla} \cdot \mathbf{u},
$$

where $\phi=1-\rho$ is the porosity, and $\mu$ is the viscosity of air. This equation is valid for compressible flows since it assumes $\rho_{a} \propto \phi P$ (isothermal ideal gas law) for the air density, $\rho_{a}$. If instead an incompressible liquid is considered this assumption becomes $\rho_{l} \propto \phi$ for the liquid density $\rho_{l}$. The pressure Eq. (2) then simplifies to a Poisson equation for the pressure given by $\boldsymbol{\nabla} \cdot[\kappa(\phi) / \mu \boldsymbol{\nabla} P]=\boldsymbol{\nabla} \cdot \mathbf{u}$. 
TABLE II. Listing of the diameters of the grains and the dimensions of the cells used in the simulations.

\begin{tabular}{lccccccc}
\hline \hline $\begin{array}{l}\text { Diameter } \\
(\mu \mathrm{m})\end{array}$ & 70 & 140 & 210 & 280 & 350 & 420 & 490 \\
\hline Width $(\mathrm{mm})$ & 28 & 56 & 84 & 112 & 140 & 168 & 196 \\
Height $(\mathrm{mm})$ & 34 & 68 & 102 & 136 & 170 & 204 & 238 \\
\hline \hline
\end{tabular}

The grains are governed by Newton's second law

$$
m \frac{d \mathbf{v}}{d t}=m g+\mathbf{F}_{\mathrm{I}}-\frac{V \boldsymbol{\nabla} P}{\rho},
$$

where $m, \mathbf{v}$, and $V$ are, respectively, the mass, velocity, and volume of a grain. The interparticle normal force $\mathbf{F}_{\mathrm{I}}$ acting between grains in contact is calculated iteratively using contact dynamics [20] (but a Hookean or Hertzian contact law may be used instead). The dynamics of the grains are simplified by neglecting particle-particle and particle-wall friction. A lower cutoff is imposed on the solid fraction because the Carman-Kozeny relation is not valid for a solid fraction less than 0.25 [21]. This cutoff causes the permeability in the most dilute regions of the system to be slightly underestimated. This leads to a slight overestimation of the pressure forces acting on grains in dilute regions. The effect of this artifact is however decreasing with increasing granular inertia.

A series of seven simulations are performed and each simulation uses monodisperse grains of different diameters, see Table II. The same relative start configuration of 160000 grains is used in all the simulations and the size of the system, given in Table II, scales proportionally to the diameters of the grains. The mass density of the grains is $2.5 \mathrm{~g} / \mathrm{cm}^{3}$, a common value for glass beads. In the simulations we have introduced a larger density than in the experiments in order to minimize the numerical artifacts caused by the solid frac-

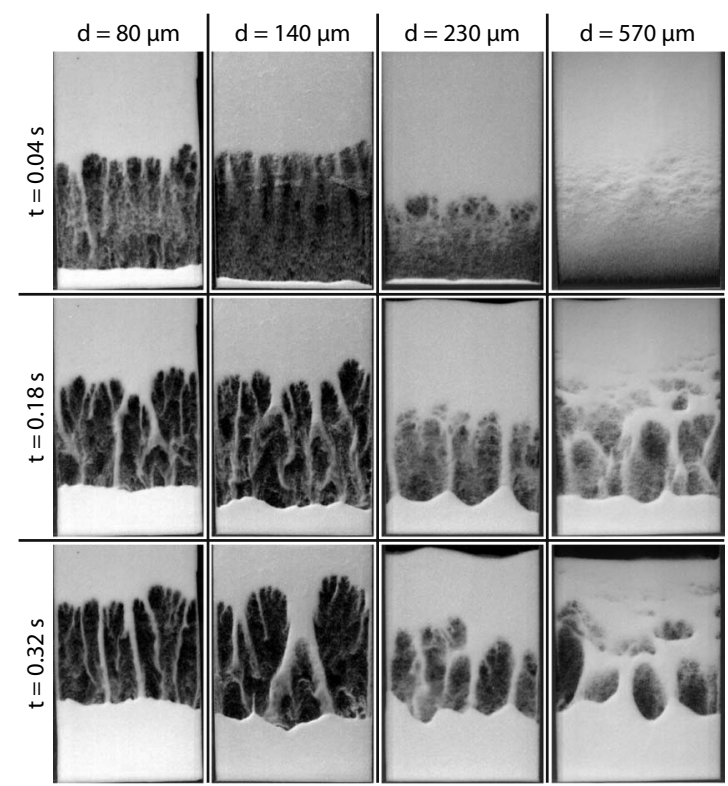

FIG. 3. Experimental snapshots. Each column represents a different experiment characterized by the diameter $d$ of the grains used, and each row represents a different time $t$.

tion cutoff. These artifacts are manifested as slightly curved and buckled fingers that become visible as the inertia of the grains decreases.

\section{RESULTS AND ANALYSIS}

Images from each of the seven simulations and four experiments are, respectively, shown in Figs. 2 and 3. The corresponding spatial dimensions of the cells are given in Tables I and II. Each row of images in Figs. 2 and 3 represents a given time $t$, and each column of images represents a given grain-diameter $d$. From the snapshots in Figs. 2 and 3 it is evident that the finger-bubble structures depend strongly on grain size. For cells with grains of diameters smaller than

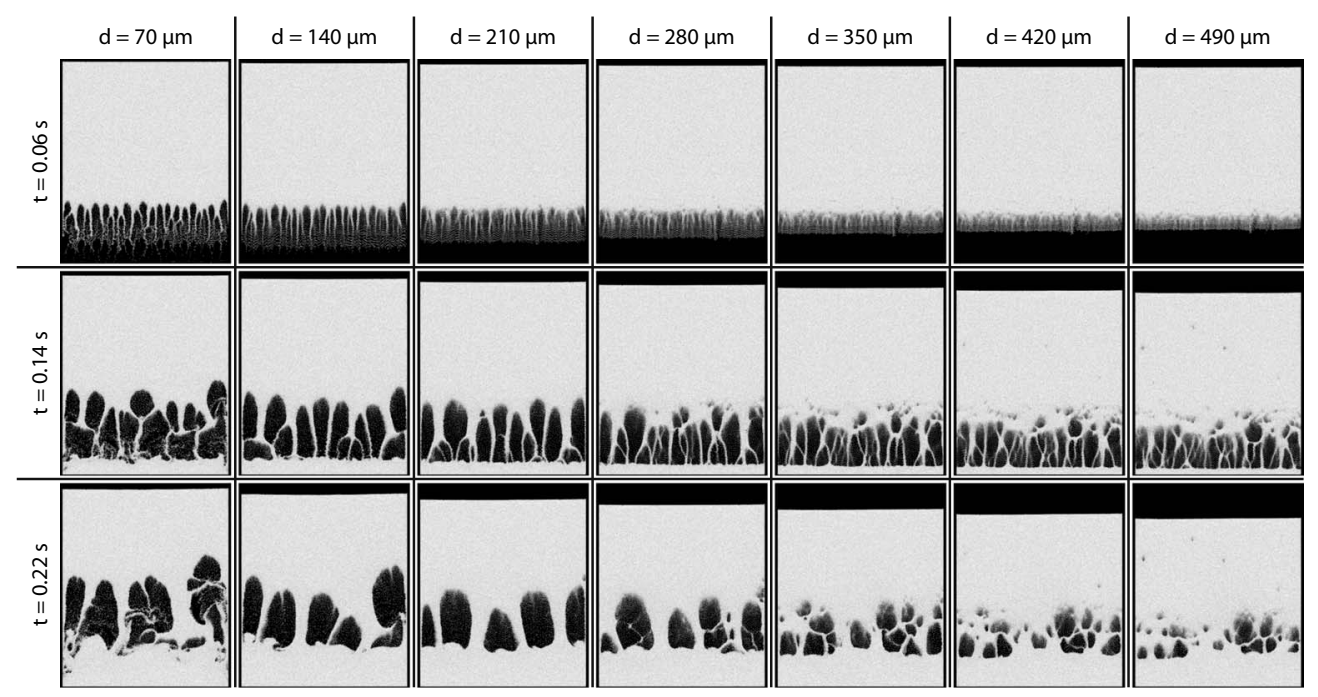

FIG. 2. Numerical snapshots. Each column represents different simulations using grains of diameter $d$, and each row represents snapshots at different times $t$. 

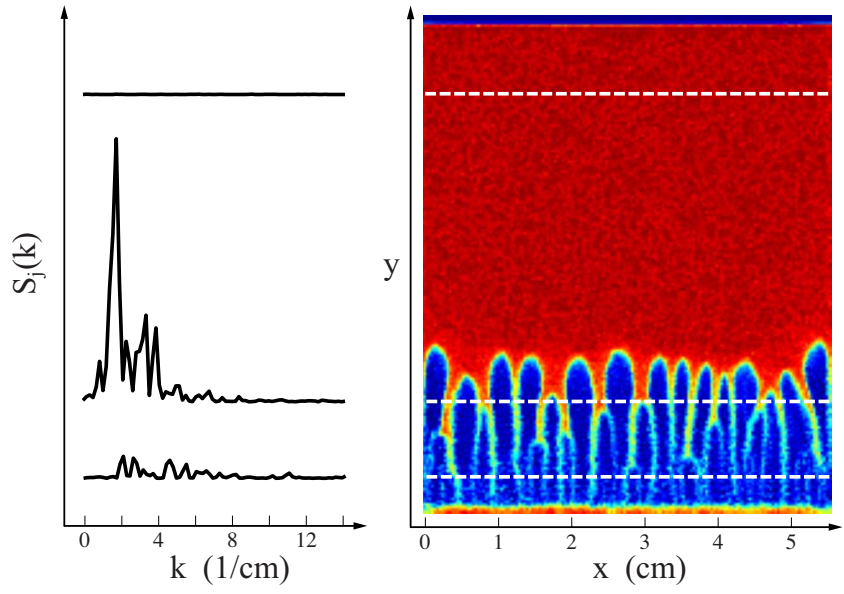

FIG. 4. (Color online) Plots of Fourier power spectra $S_{j}(k)$ (left) obtained at three different heights of the solid fraction field $\rho(x, y)$ (right) shown as a color coded data image [red (gray) is high $\rho$, blue (dark gray) is low $\rho$ ]. The stapled white lines indicate at which $y$ position the power spectra are obtained.

$200 \mu \mathrm{m}$ it is relatively easy to identify a unique interface that separates an upper, dense granular packing from the air below. For grain diameters greater than $200 \mu \mathrm{m}$, however, small air-bubbles appear in the bulk of the packing above the grain-air interface. These precursory bubbles are separated by horizontal filaments of grains which form in addition to the vertical fingers. The number of precursory bubbles increases as the grain diameter increases. These structures are analogous to the bubbles observed in the ripple instability arising in a tilted tube of sand $[16,22]$.

\section{Scale invariance}

A size measurement of the finger structures is obtained by making a Fourier transform of the solid fraction field $\rho(x, y)$. The resulting Fourier spectrum $\bar{S}(k, t)$, i.e., the wave number distribution for a given time $t$, enables a quantitative comparison of finger structures for different grain and system sizes.

The distribution $\bar{S}(k, t)$ is calculated as follows. The power spectrum $S_{j}(k)$ of each horizontal line $j$ of the solid fraction field is obtained by the discrete Fourier transform [23], using Hamming data windows to avoid frequency leakage due to the nonperiodic character of the system. By averaging over the individual power spectra $S_{j}(k)$ the final average distribution $\bar{S}(k, t)$ is obtained. This procedure is illustrated in Fig. 4: the left plot shows power spectra $S_{j}(k)$ obtained at three given positions $y_{j}$ of horizontal cuts of the density function $\rho(x, y)$ shown in the right plot of the same figure. Large $\rho$ values appear in red (gray) and small $\rho$ values appear in blue (dark gray).

As Fig. 4 illustrates it is not necessary to reduce the Fourier analysis to a spatial window centered around the interface. The power spectra obtained in the bulk above the interface are anyway flat and make no contributions to the final averaged power spectrum.
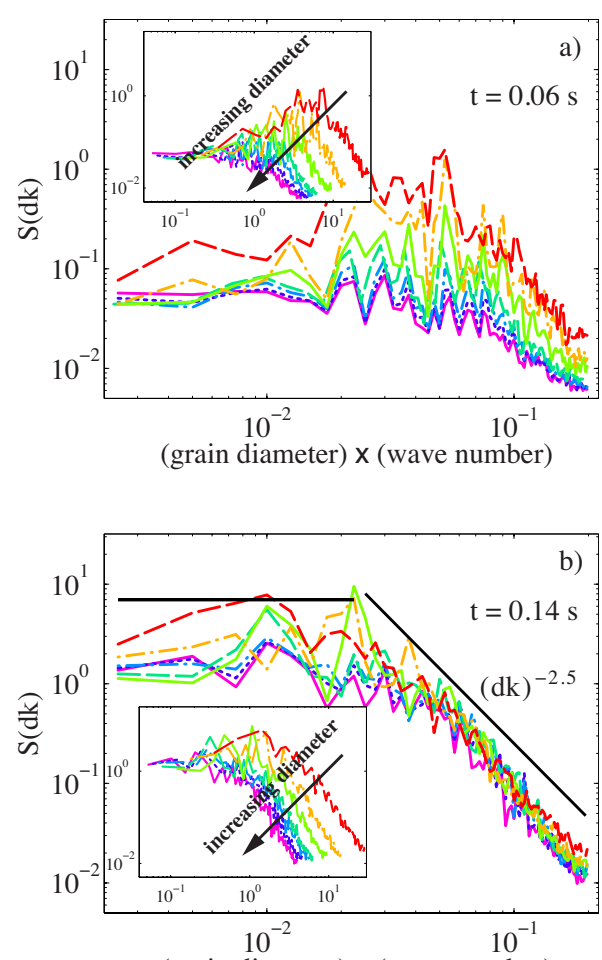

(grain diameter) $\mathrm{x}$ (wave number)

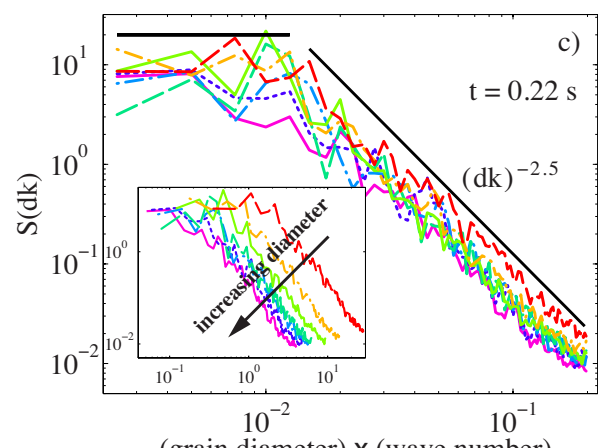

FIG. 5. (Color online) Plots of $\bar{S}(d k)[\bar{S}(k)$ in inset plots $]$ obtained from numerical data. Each plot is a data collapse of $\bar{S}(d k)$ for all grain-diameters $d$ at three different times $t$ (the same times as in Fig. 2). The different colors represent different grain diameters ranging from 70 to $490 \mu \mathrm{m}$ as indicated in the inset plots. The straight black lines are power laws obeyed by $\bar{S}(d k)$ at large and small scales: $\bar{S}(d k) \sim$ constant at large scales (growing white noise), and $\bar{S}(d k) \sim(d k)^{-2.5}$ at small scales.

The experimental data are treated similarly to the numerical data. However, the solid fraction field is not directly available in the experimental data and it is therefore estimated by the gray level values of the image pixels. The pixel value is assumed to be linearly related to the solid fraction. The inaccuracy of this assumption is not likely to have a large effect on the measurements.

Figure 5 shows $\log$-log plots of $\bar{S}(d k)$, i.e., the power spectra $\bar{S}(k)$ rescaled by the grain-diameter $d$, for the numerical data. The inset plots show the unscaled spectra $\bar{S}(k)$ as function of $k$ in $\mathrm{cm}^{-1}$. Notice that the power spectra in Fig. 5 

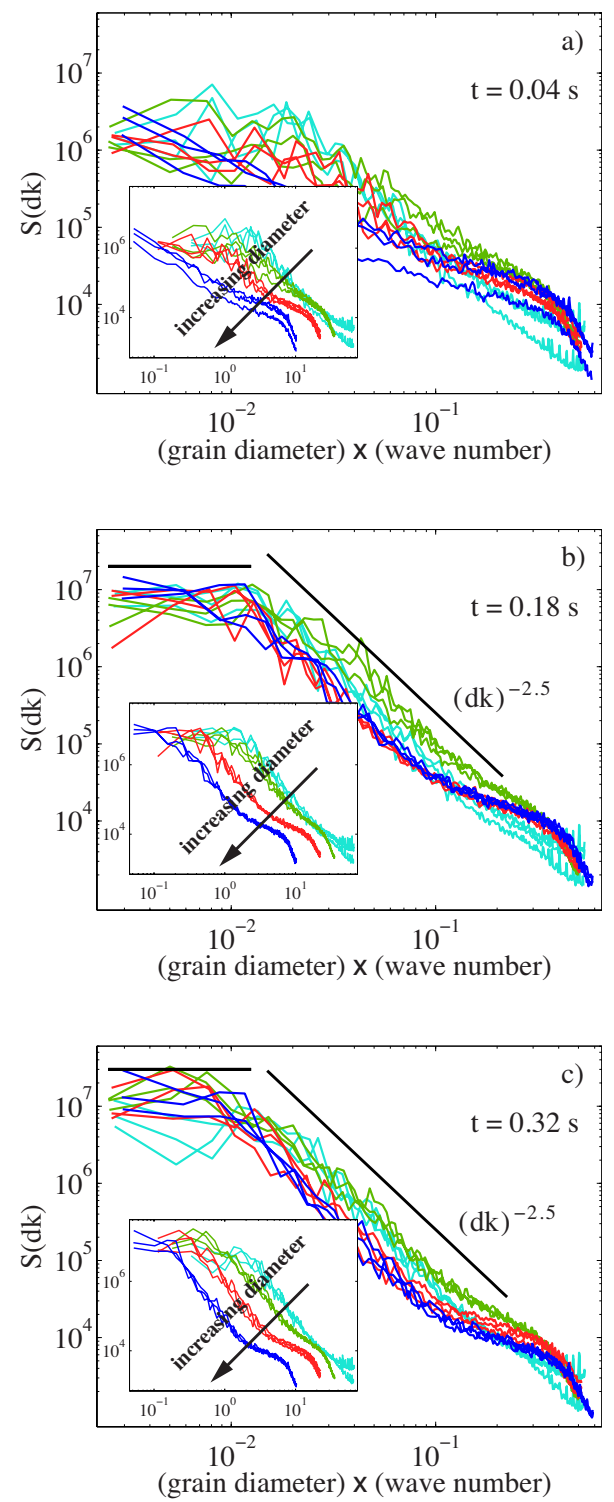

FIG. 6. (Color online) Plots of $\bar{S}(d k)[\bar{S}(k)$ in inset plots $]$ obtained from experimental data. Each plot is a data collapse of $\bar{S}(d k)$ for all grain-diameters $d$ at three different times $t$ (the same times as in Fig. 3). The different colors represent different grain diameters ranging from $80 \mu \mathrm{m}$ to $570 \mu \mathrm{m}$ as indicated in the inset plots. The straight black lines are power laws obeyed by $\bar{S}(d k)$ at large and small scales, as in the numerical case: $\bar{S}(d k) \sim$ constant at large scales, and $\bar{S}(d k) \sim(d k)^{-2.5}$ at small scales.

are calculated from the numerical data shown in Fig. 2. Figure 6 similarly shows log-log plots of $\bar{S}(d k)$ obtained from the experimental data presented in Fig. 3. The inset plots show the unscaled spectra $\bar{S}(k)$.

The plot in Fig. 5(a) hardly qualifies as a data collapse, in contrast to the other plots in Figs. 5 and 6. This is due to the fact that initially the falling grains are constantly expanding which increases the divergence of the velocity field, $\boldsymbol{\nabla} \cdot \mathbf{u}$. As is shown in Eq. (8) the source term, $P \boldsymbol{\nabla} \cdot \mathbf{u}$, of the pressure equation Eq. (2) will break the invariance. Hence, the invariance observed for larger times is only possible due to the smallness of the source term for larger times.

The characteristic wave number (where the power spectra start to decrease) seen in Fig. 5 gradually shifts from higher to lower values as time evolves. This corresponds well with the observed coarsening with time of the finger structures both in the numerical and the experimental data. However, compared to the numerical data, the shift from higher to lower wave numbers is less evident in the experimental data since the initially very fine fingers is not observed in the experiments.

After the very initial period, the (collapsed) power spectra plotted in Figs. 5 and 6 can be shown to be consistent with two power laws: $\bar{S}(d k) \sim$ constant for large scales (growing white noise), and $\bar{S}(d k) \sim(d k)^{-2.5}$ at small scales (straight black lines in Figs. 5 and 6). These exponents can be obtained from linear fits of the bilogarithmic plots, and are determined with a precision of order 0.5 . These power laws are valid both for the numerical (Fig. 5) and experimental (Fig. 6) results. In the experiments, however, there is also an additional bump of the power spectra for the smallest scales (largest wave numbers) departing from the $\bar{S}(d k) \sim(d k)^{-2.5}$ scaling behavior. This behavior may be attributed to the fact that these largest $k$ values are at the limit of the spatial resolution in the experiments.

The early-time power spectra displayed in Figs. 5(a) and 6(a) are quite scattered around the power laws. This might be related to the fact that at early times the size scaling is also not satisfied due to accelerating grains, as previously discussed.

\section{THEORETICAL INTERPRETATION}

Let us assume that we have a solution of our gas and grain equations. Now, if we magnify all physical scales, the velocities and the pressure variations by a constant factor $\lambda$, how close will we come to a solution of our equations?

In mathematical terms, let $P, \rho(\mathbf{x}, t)=m \rho_{n}(\mathbf{x}, t)$, and $\mathbf{v}_{i}$ be the pressure field, the mass density field, and particle velocities that solve the equations

$$
\begin{gathered}
\phi \frac{d P}{d t}=\boldsymbol{\nabla} \cdot\left(\frac{\kappa P \nabla P}{\mu}\right)-P \nabla \cdot \mathbf{u}, \\
m \frac{d \mathbf{v}}{d t}=m g+F_{I}-\frac{m \boldsymbol{\nabla} P}{\rho},
\end{gathered}
$$

where as before $\mathbf{u}$ is the local average of the $\mathbf{v}_{i}$ 's. We split the velocity as follows: $\mathbf{v}_{i}=\delta \mathbf{v}_{i}+\mathbf{u}_{0}$ and $\mathbf{u}=\delta \mathbf{u}+\mathbf{u}_{0}$, where $\mathbf{u}_{0}$ is the constant sedimentation velocity of a close packed system. We substitute $P=P_{0}+\delta P$ and $F_{I}=m a_{I}$ and make the observation that $\delta P \ll P_{0}$, which leads to the justified approximation

$$
\begin{gathered}
\phi \frac{\partial \delta P}{\partial t}=\boldsymbol{\nabla} \cdot\left(\frac{\kappa P_{0} \boldsymbol{\nabla} \delta P}{\mu}\right)-P_{0} \boldsymbol{\nabla} \cdot \delta \mathbf{u}, \\
\frac{d \mathbf{v}}{d t}=\mathrm{g}+a_{I}-\frac{\boldsymbol{\nabla} P}{\rho} .
\end{gathered}
$$

Here the substantial derivative has been replaced by the partial derivative because $\mathbf{u} \cdot \boldsymbol{\nabla} \delta P \ll P_{0} \boldsymbol{\nabla} \cdot \delta \mathbf{u}$. A rough estimate 
using $u \sim 10^{-2} \mathrm{~m} / \mathrm{s}, \nabla \delta P \sim 10^{-3} \mathrm{~Pa} / \mathrm{m}, P_{0}=10^{5} \mathrm{~Pa}$, and $\boldsymbol{\nabla} \cdot \delta \mathbf{u} \sim 10 \mathrm{~s}^{-1}$ justifies this assumption. As in a hydrostatic system we assume that the pressure field of the magnified system is $\delta P^{\prime}\left(\mathbf{x}^{\prime}, t\right)=\lambda \delta P(\mathbf{x}, t)=\lambda \delta P\left(\mathbf{x}^{\prime} / \lambda, t\right)$, where $\mathbf{x}^{\prime}=\lambda \mathbf{x}$. We make the following scaling ansatz, expressing the solutions of Eqs. (5) in terms of the scaled fields as

$$
\begin{aligned}
\delta P(\mathbf{x}, t) & =\frac{1}{\lambda} \delta P^{\prime}\left(\mathbf{x}^{\prime}, t\right), \\
\mathbf{u}_{0} & =\frac{1}{\lambda^{2}} \mathbf{u}_{0}^{\prime}, \\
\delta \mathbf{u}(\mathbf{x}, t) & =\frac{1}{\lambda} \delta \mathbf{u}^{\prime}\left(\mathbf{x}^{\prime}, t\right), \\
\rho(\mathbf{x}, t) & =\rho^{\prime}\left(\mathbf{x}^{\prime}, t\right),
\end{aligned}
$$

where the sedimentation velocity $\mathbf{u}_{0}^{\prime}$ scales as $\lambda^{2}$ because the local density is $\lambda$-invariant and permeability goes as length squared and scales as $\lambda^{2}$, i.e.,

$$
\kappa^{\prime}=\lambda^{2} \kappa .
$$

Note that Eqs. (5) are unaffected by the scaling of $\mathbf{u}_{0}$. Using the new length scale in the derivative $\nabla^{\prime}$ we get $\boldsymbol{\nabla}=\lambda \boldsymbol{\nabla}^{\prime}$. The pressure gradient $\nabla \delta P, \rho, \phi$, and $t$ are all invariant. By substitution we obtain

$$
\begin{aligned}
\phi \frac{\partial \delta P^{\prime}}{\partial t}= & \nabla^{\prime} \cdot\left(\frac{\kappa^{\prime} P_{0} \nabla^{\prime} \delta P^{\prime}}{\mu}\right)-\lambda P_{0} \nabla^{\prime} \cdot \delta \mathbf{u}^{\prime}, \\
& \frac{1}{\lambda}\left(\frac{d \mathbf{v}^{\prime}}{d t}-a_{I}^{\prime}\right)=\mathrm{g}-\frac{\boldsymbol{\nabla}^{\prime} P^{\prime}}{\rho^{\prime}},
\end{aligned}
$$

as the equations satisfied by the scaled fields. Note that by mass conservation of the granular phase, i.e., $d \rho / d t=-\rho \boldsymbol{\nabla} \cdot \delta \mathbf{u}$, the last term of the pressure equation may be written $-P_{0} \boldsymbol{\nabla} \cdot \delta \mathbf{u}=\left(P_{0} / \rho\right) d \rho / d t$, and this term thus gives the effect of density changes in a frame of reference moving with the grains. This implies that the term vanishes for a flow field without internal compression or expansion. If such a flow field is also steady, like that of a slab of connected particles moving at a constant velocity, the acceleration terms vanish along with the $-P_{0} \boldsymbol{\nabla} \cdot \delta \mathbf{u}$ term, i.e., all the $\lambda$-dependent terms vanish in Eq. (8). This invariance means that the scaled fields are solutions of the same equations as the original fields.

The terms that are multiplied by a $\lambda$-factor break the invariance. To get an estimate of their relative magnitude we introduce dimensionless numbers. Taking the characteristic length scale of the flow to be $l$ and the time scale to be $l / u$ we may write

$$
\mathrm{Fr}=\frac{u^{2}}{g l} \sim \frac{d \mathbf{v} / d t}{g},
$$
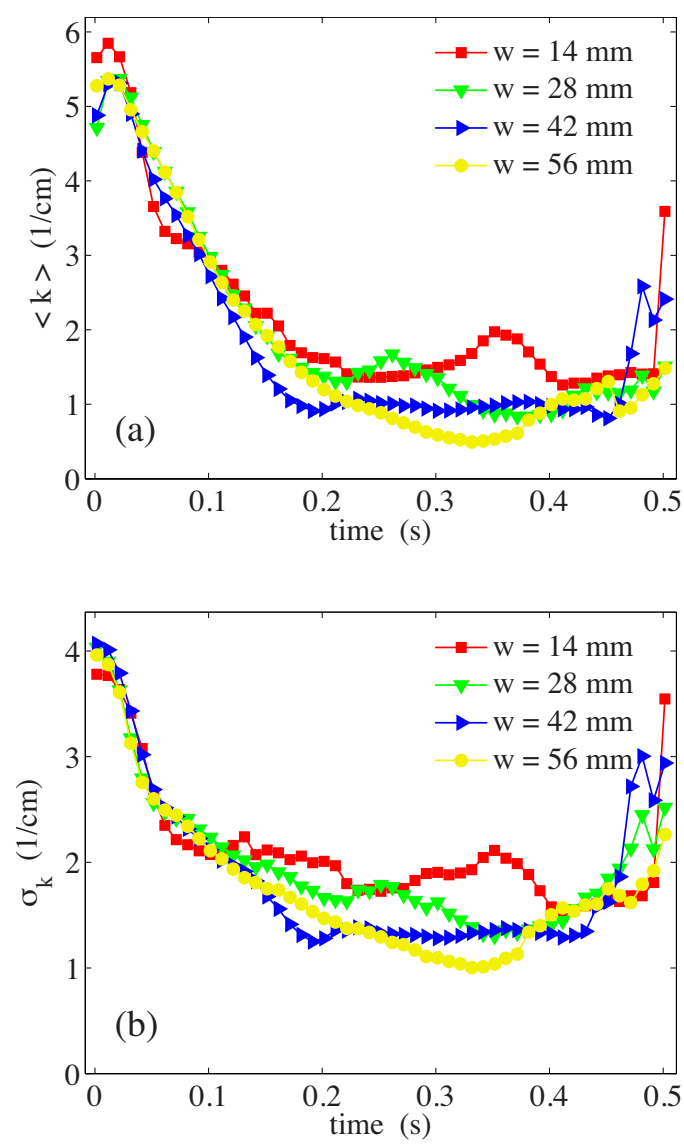

FIG. 7. (Color online) (a) Plot of the mean wave number $\langle k\rangle$ obtained from four different simulations with grains of diameter $d=140 \mu \mathrm{m}$ but with four different cell widths $w$. (b) Same plot for the standard deviation $\sigma$.

$$
A=\frac{180 \mu u l}{\delta P d^{2}} \sim \frac{P_{0} \boldsymbol{\nabla} \cdot \delta \mathbf{u}}{\boldsymbol{\nabla} \cdot\left(\frac{\kappa P_{0} \boldsymbol{\nabla} \delta P}{\mu}\right)},
$$

where $\mathrm{Fr}$ is known as the Froude number. Taking $d=140 \mu \mathrm{m}, \quad l=\langle k\rangle^{-1}=0.01 \mathrm{~m} \quad$ at $t=0.5 \mathrm{~s} \quad$ and $u=l / t=0.02 \mathrm{~m} / \mathrm{s}$ we get $\mathrm{Fr}=0.004$. With $\delta P=0.01 \mathrm{~atm}$. and the same values for the length scale $l$ we get $A=0.04$.

Since $A$ and Fr measure the relative magnitude of the invariance-breaking terms, the smallness of $A$ and Fr indicate that the scaled fields are close to satisfying the physical Eqs. (5). For this reason we may expect all lengths in the simulations to scale the same way, and $\bar{S}(d k)$ will be invariant under scaling by $\lambda$. However, since $\operatorname{Fr} \propto \lambda$, increasing the magnification will increase the relative effect of granular inertia, and, we expect, the deviations from this scaling property. On the other hand, $A \propto 1 / \lambda$ so decreasing the particle size will cause the compression term in the pressure equation to grow in relative magnitude. It is therefore only in a certain window of particle sizes around $d=140 \mu \mathrm{m}$ that we may expect the scale invariance. In particular, for the $d=70 \mu \mathrm{m}$ simulations it is likely that deviations from the scaling behavior arise due to the relatively large $A$ value.

The above scaling invariance is somewhat analogous to the scale invariance of low Reynolds number flows, where 
the inertial term may be neglected since it is small compared to the viscous term, and symmetries of the flow solutions emerge because the Navier-Stokes equations are replaced by the Stokes equation.

\section{CONTAINER SIZE EFFECTS}

In the experiments and simulations presented so far the size of the grains and the size of the container are changed proportionally to each other such that every container confines approximately the same number of grains. To address the question of possible container size effects a series of simulations is performed where the size of the grains and the size of the container are changed independently. Four different simulation geometries are used while the grain diameter is kept fixed at $140 \mu \mathrm{m}$. The four numerical cells all have the same height of $68 \mathrm{~mm}$ but different widths of 14, 28, 42, and $56 \mathrm{~mm}$. To compare the different results the power spectrum $\bar{S}(k, t)$ for each of the four simulations is obtained as explained in Sec. IV. In order to represent the temporal evolution of $\bar{S}(k, t)$ the mean and standard deviation of $\bar{S}(k, t)$ are calculated by the usual definitions

$$
\begin{gathered}
\langle k\rangle(t)=\frac{\sum k \bar{S}(k, t)}{\sum \bar{S}(k, t)}, \\
\sigma(t)=\sqrt{\frac{\sum k^{2} \bar{S}(k, t)}{\sum \bar{S}(k, t)}-\langle k\rangle(t)^{2}},
\end{gathered}
$$

where the summation is over all $k$ values.

Figure 7 shows the mean wave number $\langle k\rangle$ and standard deviation $\sigma$ for the four different geometries. During the coarsening stage, i.e., from $t=0 \mathrm{~s}$ until $t=0.2 \mathrm{~s}$, the four curves overlap quite well. For $t>0.2 \mathrm{~s}$ the curves are more spread due to two peaks in the $w=14$ and $28 \mathrm{~mm}$ data. These peaks are most likely caused by fluctuations in the evolving structures which are not averaged out due to less statistics for the narrow geometries. In addition, the different granular configurations used in the four runs also account for some variations in the data. For cell widths less than roughly 14 $\mathrm{mm}$ container size effects will be present since this width is smaller than the maximum width a bubble may reach in a wide cell. However, the data in Fig. 7 shows no systematic dependence on the container size.

In [10] it was shown that plots of $\langle k\rangle$ for different grain sizes will overlap only if $\langle k\rangle$ is rescaled by the graindiameter $d$. In Fig. 7 the plots overlap although no rescaling of $\langle k\rangle$ by, e.g., the container width $w$ is applied. Hence, the observed pattern scales with grain size and not container size.

\section{CONCLUSION AND SUMMARY}

In summary we have uncovered an approximate scale invariance in a granular Rayleigh-Taylor instability $[10,11]$ through experimental measurements and complementary simulations. In addition, a theoretical interpretation is provided with the conclusion that the validity of the scale invariance is limited to a window of grain diameters from roughly $70 \mu \mathrm{m}$ to about $570 \mu \mathrm{m}$. The scale invariance may be interpreted as the existence of a Stokes-like regime for the investigated systems. Terms arising from grain inertia for large grains, or from pressure sources (i.e., $P \boldsymbol{\nabla} \cdot \mathbf{u}$ ) for small grains, will gradually break the invariance. A separate series of simulations with constant grain sizes but different geometries was performed with the conclusion that the observed scale invariance is not affected by container size effects. The deviations observed in this invariance has been given interpretations both in terms of theoretical arguments and in terms of numerical and experimental imperfections.
[1] Physics of Dry Granular Media, edited by H. J. Herrmann, J.-P. Hovi, and S. Luding, NATO ASI Series E: Applied Sciences Vol. 350 (Kluwer Academic Publishers, Dordrecht, 1998).

[2] G. K. Batchelor, J. Fluid Mech. 52, 245 (1972).

[3] J. F. Richardson and W. N. Zaki, Trans. Inst. Chem. Eng. 3, 65 (1954).

[4] P. Y. Julien, River Mechanics (Cambridge University Press, Cambridge, 2002)

[5] D. H. Rothman, J. P. Grotzinger, and P. B. Flemings, J. Sediment Res. 64, 59 (1994).

[6] R. Bachrach, A. Nur, and A. Agnon, J. Geophys. Res. 106, 13515 (2001).

[7] J. F. Davidson and D. Harrison, Fluidization (Academic Press, New York, 1971).

[8] D. Gidaspow, Multiphase Flow and Fluidization (Academic Press, San Diego, 1994).

[9] G. Taylor, Proc. R. Soc. London, Ser. A 201, 192 (1950).

[10] J. L. Vinningland, Ø. Johnsen, E. G. Flekkøy, R. Toussaint, and K. J. Måløy, Phys. Rev. Lett. 99, 048001 (2007).

[11] J. L. Vinningland, Ø. Johnsen, E. G. Flekkøy, R. Toussaint, and K. J. Måløy, Phys. Rev. E 76, 051306 (2007).

[12] C. Völtz, W. Pesch, and I. Rehberg, Phys. Rev. E 65, 011404 (2001).

[13] Microbeads AS, P. O. Box 265, N-2021 Skedsmokorset, NORWAY, Tel: +47 648353 00, Fax: +47 648353 01, email: support@micro-beads.com.

[14] S. McNamara, E. G. Flekkøy, and K. J. Måløy, Phys. Rev. E 61, 4054 (2000).

[15] D.-V. Anghel, M. Strauss, S. McNamara, E. G. Flekkøy, and K. J. Måløy, Phys. Rev. E 74, 029906(E) (2006).

[16] E. G. Flekkøy, S. McNamara, K. J. Måløy, and D. Gendron, Phys. Rev. Lett. 87, 134302 (2001).

[17] Ø. Johnsen, R. Toussaint, K. J. Måløy, and E. G. Flekkøy, Phys. Rev. E 74, 011301 (2006).

[18] H. Darcy, Les Fontaines Publiques de la Ville de Dijon (Dalmont, Paris, 1856).

[19] P. C. Carman, Trans. Inst. Chem. Eng. 15, 150 (1937). 
[20] F. Radjai, M. Jean, J.-J. Moreau, and S. Roux, Phys. Rev. Lett. 77, 274 (1996).

[21] A. A. Zick and G. M. Homsy, J. Fluid Mech. 115, 13 (1982).

[22] D. Gendron, H. Troadec, K. J. Måløy, and E. G. Flekkøy, Phys. Rev. E 64, 021509 (2001).
[23] W. H. Press, S. A. Teukolsky, W. T. Vetterling, and B. P. Flannery, Numerical Recipes in $\mathrm{C}++$ : The Art of Scientific Computing (Cambridge University Press, Cambridge, 2002), p. 505, Chap. 12. 